\title{
High-throughput sequencing of African chikanda cake highlights conservation challenges in orchids
}

\author{
Sarina Veldman ${ }^{1} \cdot$ Barbara Gravendeel $^{2,3} \cdot$ Joseph N. Otieno $^{4} \cdot$ \\ Youri Lammers ${ }^{2} \cdot$ Elza Duijm $^{2}$ - Aline Nieman ${ }^{2}$. \\ Benny Bytebier ${ }^{5} \cdot$ Grace Ngugi $^{5}$ - Florent Martos ${ }^{5}$. \\ Tinde R. van Andel ${ }^{2,6}$ • Hugo J. de Boer ${ }^{1,2,7}$ (D)
}

Received: 14 July 2016/Revised: 18 March 2017/Accepted: 30 March 2017/

Published online: 1 April 2017

(C) The Author(s) 2017. This article is an open access publication

\begin{abstract}
Chikanda is a traditional dish made with wild-harvested ground orchid tubers belonging to three orchidioid genera, Disa, Satyrium and Habenaria, all of which are CITES appendix II-listed. Identification of collected orchid tubers is very difficult and documentation of constituent species in prepared chikanda has hitherto been impossible. Here amplicon metabarcoding was used in samples of six prepared chikanda cakes to study genetic sequence diversity and species diversity in this product. Molecular operational taxonomic unit identification using similarity-matching reveals that species of all three genera were present in the chikanda samples studied. Disa was present in all of the samples, Satyrium in five out of six and Habenaria in one of the samples, as well as a number of other plants. The fact that each sample contained orchids and the presence of a wide variety of species from all genera in this traditional dish raise serious concerns about the sustainability of this trade and the future of wild orchid populations in the main harvest areas. This proof-of-concept study shows that Ion-Torrent PGM is a cost-effective scalable platform for metabarcoding using the relatively long nrITS1 and nrITS2 regions.
\end{abstract}

Communicated by Daniel Sanchez Mata.

Electronic supplementary material The online version of this article (doi:10.1007/s10531-017-1343-7) contains supplementary material, which is available to authorized users.

Hugo J. de Boer

hugo.deboer@nhm.uio.no

1 Department of Organismal Biology, Uppsala University, Norbyvägen 18D, 75236 Uppsala, Sweden

2 Naturalis Biodiversity Center, Darwinweg 2, 2333 CR Leiden, The Netherlands

3 University of Applied Sciences Leiden, Leiden, The Netherlands

4 Institute for Traditional Medicine, Muhimbili University of Health and Allied Sciences, Dar es Salaam, Tanzania

5 School of Life Sciences, University of KwaZulu-Natal, Pr. Bag X01, Scottsville 3209, South Africa

6 Wageningen University and Research, Wageningen, The Netherlands

7 Natural History Museum, University of Oslo, P.O. Box 1172, Blindern, 0318 Oslo, Norway 
Furthermore, nrITS metabarcoding can be successfully used for the detection of specific ingredients in a highly-processed food product at genus level, and this makes it a useful tool in the detection of possible conservation issues arising from commercialized trade or processed plant products.

Keywords CITES · Disa · Ethnobotany · Habenaria $\cdot$ Ion-Torrent PGM - Satyrium . Wildlife forensics

\section{Introduction}

Chikanda is a traditional dish that consists primarily of wild-harvested terrestrial orchid tubers and peanuts (Davenport and Ndangalasi 2001). This meatloaf-like dish is prepared by mixing ground orchid tubers with peanut flour, boiling and thickening the mixture in water and subsequent baking (Veldman et al. 2014). For many years, ethnic groups in northeastern Zambia and in adjacent provinces in Tanzania, the Democratic Republic of Congo and Malawi have been known to consume chikanda in times of famine or as a seasonal addition to their dietary staples (Richards 1939; Cribb and Leedal 1982; Bingham and Smith 2002; Davenport and Ndangalasi 2003; Kasulo et al. 2009). Over the past decades, however, chikanda has gained popularity throughout the country as a nutritious snack. Whereas tubers used to be harvested on a household scale and presumably without the sustainability of local orchid populations being affected, they are now commercially harvested in large amounts to meet increasing demand (Davenport and Ndangalasi 2001; Bingham 2004). As a result, Zambian orchid resources are declining rapidly, and traders are driven to obtain their tubers from further afield (Davenport and Ndangalasi 2003; Bingham 2004; Veldman et al. 2014). The majority of commercialized tubers in the main market of Zambia's Capital City Lusaka originate from the Southern Highlands of Tanzania (Bingham 2004). In addition to this, significant quantities of the tubers are now also coming from Angola, the Democratic Republic of the Congo, Malawi and Mozambique (Veldman et al. 2014). Despite the fact that all orchids are CITES appendix II listed (2014), and thus their international trade is subject to specific rules and permits, an estimated 2.2-4.0 million orchid tubers are illegally exported from Tanzania to Zambia each year, and a total of 85 terrestrial orchid species are identified to be at risk of overharvesting (Davenport and Ndangalasi 2003; Veldman et al. 2014). Inevitably, this enormous pressure on Tanzanian orchid populations is unsustainable, and Tanzanian collectors are increasingly forced to look for alternative collection sites further away (Davenport and Ndangalasi 2003; Nyomora 2005; Challe and Price 2009).

The orchids used for chikanda are mainly species belonging to three terrestrial orchid genera: Disa, Satyrium and Habenaria (Cribb and Leedal 1982; Bingham and Smith 2002; Davenport and Ndangalasi 2003). Challe and Price (2009) show that chikanda collectors preferentially collect Disa robusta, followed by Satyrium trinerve (syn: Satyrium atherstonei) and Habenaria xanthochlora. However, previous preferences for specific terrestrial orchid species are no longer apparent since collectors have started to harvest tubers indiscriminately (Bingham 2004). Other sources corroborate a preference for the genus Disa and specifically for wetland species of this genus (Davenport and Ndangalasi 2001; Nyomora 2005; Hamisy 2008; Challe and Price 2009). Habenaria and Satyrium species are also mentioned, but they are considered to be of inferior quality (Richards 1939; Davenport 
and Ndangalasi 2001; Bingham 2004; Nyomora 2005; Hamisy 2008; Challe and Price 2009). Recent surveys, however, have shown that more rare, dryland and forest species are now harvested because of local scarcity, as well as species from the genera Brachycorythis (Hamisy 2008), Eulophia (Hamisy 2008) and Roeperocharis (Hamisy 2008; Challe and Price 2009).

Species-level identification of the tubers that are being used for chikanda is a major challenge, and it remains unclear exactly which species are collected. This is mainly caused by a lack of distinctive morphological characteristics of the tubers and is exacerbated as most are harvested after the flowering season, leaving few or no fertile characters for specimen identification in the field. Moreover, once the tubers enter the market chain, it becomes impossible to discriminate between tubers of different orchid species, and even attempting to distinguish them at the genus level is prone to errors (Davenport and Ndangalasi 2003). Collectors and traders of orchid tubers for chikanda are known to have various local classifications of these tubers based on vernacular names. Collectors distinguish real chikanda tubers that yield a solid, gelatinous texture, and msekelele chikanda tubers that yield a crumbling, coarser product that is considered of inferior quality. Another way for people to distinguish between the tubers is based on the type of soil from which they were harvested (Davenport and Ndangalasi 2003). However, several problems might occur when these local classifications are to be translated into scientific classifications (Otieno et al. 2015). In previous studies tubers were either collected for propagation to allow for the identification of flowering specimens (Nyomora 2005), or species assessments were made by accompanying collectors in the field during the flowering season and asking them to point out which species are considered edible (Davenport and Ndangalasi 2003; Challe and Price 2009). These methods can be effective, but they are time consuming and capture only the diversity of species collected by people in selected study areas.

The use of DNA barcoding and metabarcoding for the identification of commercialized plant products has evolved with advances in molecular biology and sequencing (Coghlan et al. 2012; Kool et al. 2012; Cheng et al. 2014; de Boer et al. 2014). Metabarcoding combines DNA barcoding and high-throughput sequencing (Taberlet et al. 2012). Most metabarcoding studies focusing on plants have used the plastid trnL P6 loop, as it has high primer universality, short amplicon length, high stability and high sequence variation. The combination of these characteristics has made trnL P6 the marker of choice for ancient DNA and ancient sediment DNA metabarcoding studies (Jørgensen et al. 2012; Parducci et al. 2012; Taberlet et al. 2012; Boessenkool et al. 2014; Willerslev et al. 2014). The use of nrITS1 and nrITS2 has been limited due to the shorter read length of previous highthroughput sequencing platforms, but the markers are more variable and have a greater potential for identification at finer taxonomic scales than the shorter and less variable trnL P6 loop. The higher level of sequence variation is important for identification of orchids that often have limited interspecific variation (van der Niet et al. 2005; Bytebier et al. 2007; van der Niet and Linder 2008).

Several metabarcoding studies have been published that use nrITS1 or nrITS2 for the identification of fungi (Epp et al. 2012; Blaalid et al. 2013; Schmidt et al. 2013; De Beeck et al. 2014). So far the only plant studies to use nrITS in metabarcoding have used nrITS2 to determine the taxonomic composition of pollen collected by honey bees (Galimberti et al. 2014; Richardson et al. 2015) and species composition in herbal medicines (Cheng et al. 2014; Ivanova et al. 2016).

This study takes a novel approach by focusing on nrITS metabarcoding of a highly processed and complex product: prepared orchid chikanda. DNA barcoding for species 
identification has so far not been attempted in African orchid trade, and identification of the constituent species in prepared chikanda has hitherto been impossible. Species level identification of orchid species used in prepared chikanda would allow us to identify the species that are targeted most, detect the presence of rare, threatened or narrow endemics, and enable us to identify priority species for conservation efforts. The objectives of this study were to provide useful and accurate assessments of which orchid genera are included in chikanda, while also producing useful estimates of species diversity in these samples.

We hypothesize that nrITS metabarcoding of chikanda cake will allow species level identification of the species used in chikanda cake. If this approach proves to be suitable we further hypothesize that this data can provide useful input to determining which species might be at risk of overharvesting and which species should be prioritized for conservation. This method may also be applicable to the analysis of the ingredients in other processed plant products.

\section{Materials and methods}

Research was conducted in the Southern Highlands of Tanzania in the Mbeya, Njombe and Sumbawanga regions. The mountainous grasslands of the Southern Highlands have been long recognized for their floral diversity and high level of endemism (Hedberg 1957; Cribb and Leedal 1982; Davenport and Ndangalasi 2003). The area is home to over 85 terrestrial orchid species, of which at least 31 are national or near-national endemics. The area, and in particular the Kitulo Plateau, has been under continuous harvesting pressure by chikanda tuber collectors for several decades (Davenport and Ndangalasi 2003). In 2004, the Kitulo Plateau was established as a national park with the specific aim of protecting its rich botanical biodiversity. Although this has not led to a complete stop of orchid harvesting from Kitulo, much less harvesting is reported to now take place in the protected area (Davenport and Bytebier 2004).

Surveys at local markets in Tunduma at the Tanzania-Zambia border were carried out February and September 2013. Vendors were interviewed informally about the seasonality of the trade and additional information about chikanda availability. February and September are outside the harvesting season for chikanda tubers, but during in February we found five people on either side of the border selling unprocessed orchid tubers, prepared chikanda or both. In September no vendors were observed selling ready-made chikanda and only three market vendors were selling unprocessed orchid tubers. Informal interviews with informants involved in the chikanda trade revealed that in February only lesser quality msekelele tubers were available, and in September supply by the collectors was limited to once a week or fortnight. They indicated that the peak-season for chikanda was in JuneJuly, when they receive new chikanda tuber supplies every other day.

In February, ready-made chikanda samples were purchased at the market from three different vendors (chikanda 1-3), and in September we organized chikanda to be prepared on location by two local cooks using tubers bought in the market (chikanda 4-6). Two chikanda cakes were made with tubers purchased as real chikanda (chikanda 4 and 6), and a third was made with msekelele chikanda tubers (chikanda 5). Small pieces of prepared chikanda were collected in silica gel for DNA extraction and entire vouchers were stored in $70 \%$ EtOH. Additional information, about availability of the tubers and preferred harvesting time, was collected through informal interviews in Swahili with 14 informants 
involved in chikanda collection or trade in Tunduma and Sumbawanga in Tanzania, and Nakonde and Chihanga in Zambia.

Samples were stored at room temperature prior to DNA extraction. To reduce the risk of contamination, the outer layer of each chikanda sample was removed before homogenization. Samples were homogenized using a Retsch mill (Retsch GmbH, Germany). DNA was extracted from the homogenized samples using two modified standard methods: silicabased extraction (Rohland and Hofreiter 2007) and CTAB extraction (Doyle and Doyle 1987). In the CTAB extractions, polysaccharides from the starch-rich orchid tubers formed a gelatinous substance during the CTAB-incubation step, and this gel caused difficulties during subsequent mixing with the chloroform-isoamyl alcohol and resulted in a relatively low yield of DNA from the water phase after centrifugation. In silica-based extractions, unprocessed tuber samples also formed a gel in the extraction buffer, which resulted in a low yield of DNA from the extraction. DNA purification was performed using a QIAquick PCR purification kit (Qiagen NV, The Netherlands). DNA concentration and purity were measured using a NanoDrop (ThermoFischer Scientific, USA). Extracted DNA yields ranged from 2.1 to $102.4 \mathrm{ng} / \mu \mathrm{l}$ and quality values varied between 1.2 and 2.44 (260/280 ratio).

Amplicons were generated from the nuclear ribosomal internal transcribed spacers nrITS1 and nrITS2 using primers 17SE and 5.8I1, and 5.8I2 and 26SE, respectively (Sun et al. 1994). PGM forward primers were labeled with unique -MIDx tags and reverse primers with uniform -trP1 tags. Thermal cycling was carried out in $25 \mu \mathrm{l}$ reaction volumes, and each reaction contained $5 \mu$ Phire $5 \times$ PCR buffer, $5 \mu l 5 \times$ TBT-PAR (Samarakoon et al. 2013), $0.25 \mu \mathrm{M}$ of each primer, $1 \mu \mathrm{l}$ of $10 \mathrm{mM} \mathrm{dNTP}, 0.5 \mu \mathrm{l}$ of $4 \mathrm{U} / \mu \mathrm{l}$ Phire hsTaq polymerase, $8.5 \mu \mathrm{l}$ of milliQ (Ultrapore) $\mathrm{H}_{2} \mathrm{O}$ and $3 \mu \mathrm{l}$ of template DNA. The following PCR protocol was used: $40 \mathrm{~s}$ of initial denaturation at $98{ }^{\circ} \mathrm{C}$, followed by 35 cycles of $98{ }^{\circ} \mathrm{C}$ for $5 \mathrm{~s}, 50{ }^{\circ} \mathrm{C}$ for $20 \mathrm{~s}, 72{ }^{\circ} \mathrm{C}$ for $15 \mathrm{~s}$, followed by a final extension step of $60 \mathrm{~s}$ at $72^{\circ} \mathrm{C}$. After PCR amplicons were cleaned and selected with $0.9 \times$ Agencourt AMpure beads following standard protocol. An equimolar pool concentration of these eluted amplicons was made through measurement on a Bioanalyser using a DNA 1000 chip (Agilent). Equimolar pools were then measured on the Bioanalyser with a high sensitivity chip and diluted according to the calculated template dilution factor to target 10-30\% of all positive ISPs. The template preparation of this pool was carried out on an Ion One Touch instrument with the Ion PGM Template OT2 400 kit (Life Technologies) according to manual 7218 v3.0. The ion sphere quality control kit was used to check quality of the Ion One Touch2 400 ion sphere particles on a Life Qubit 2.0. The ion sphere's were loaded on a 314 chip v2 and sequenced with the ion PGM sequencing 400 kit (Life Technologies) on an Ion-Torrent personal genome machine (Life Technologies, ThermoFischer Scientific, USA).

Sequencing reads were processed using the HTS-barcode-checker pipeline (Lammers et al. 2014) available as a Galaxy pipeline at the Naturalis Biodiversity Center (http://145. 136.240.164:8080/). Using the HTS pipeline, adapters were removed, and ITS1 and ITS2 primer sequences were used to demultiplex the sequencing reads per sample and to filter out reads that did not match any of the primers. PRINSEQ (Schmieder and Edwards 2011) was used to determine filtering and trimming values based on read lengths and Phred read quality (Ewing and Green 1998). All reads with a mean Phred quality score of less than 25 were filtered out, as well as reads with a length of less than $200 \mathrm{bp}$. Remaining reads were trimmed to a maximum length of $360 \mathrm{bp}$. CD-HIT-EST (Li and Godzik 2006) was used to cluster reads into molecular operational taxonomic units (MOTUs) defined by a sequence similarity of $>99 \%$ and a minimum number of 2 reads. The consensus sequences of non- 
singleton MOTUs were queried using BLAST (Altschul et al. 1990) against a local copy of the NCBI/GenBank nucleotide database, with a maximum e-value of 0.05 , a minimum hit length of $100 \mathrm{bp}$ and sequence identity of $>97 \%$. The number of reads per MOTU as well as the BLAST results per MOTU were compiled using custom scripts from the HTS Barcode Checker pipeline (Lammers et al. 2014). A separate identification run was performed with the complementary Habenaria dataset, to detect the presence of African Habenaria species.

A sequence reference database was compiled from all publicly available nuclear internal transcribed spacer (nrITS) sequences from the online repository NCBI GenBank, including numerous sequences of the chikanda orchid genera Disa (149 accessionscorresponding to 129 species, including 13 of the 29 Disa species occurring in Tanzania), Satyrium (127 accessions-corresponding to 63 species, including 27 of the 36 Satyrium species occurring in Tanzania) and Habenaria (335 accessions-corresponding to 100 species, of which most are from the Neotropics and 9 from Africa), as well as accessions from several additional orchid genera suspected to be used in chikanda. Since most of the nrITS sequences in NCBI GenBank for Habenaria belonged to Neotropical species the database was complemented with 88 as yet unpublished nrITS sequences of African Habenaria species that form part of the $\mathrm{PhD}$ research of G. Ngugi from the Bytebier Lab (corresponding to 61 species, including 29 of the 96 Habenaria species occurring in Tanzania). To determine whether species in these genera could be identified using MOTUs, the interspecific genetic distance variation of the three genera was analyzed using SpeciesIdentifier v 1.7.9 (Meier et al. 2006). Prior to analysis with SpeciesIdentifier, the sequences were automatically aligned using MAFFT (Katoh et al. 2002) as implemented in AliView (Larsson 2014), and only critically checked GenBank submissions, which were submitted by renowned orchid experts, were kept for analysis. The weighted homopolymer ratio (WHR) was calculated to determine if sequencing errors, which are frequent in long homopolymer regions, would be likely to lead to misidentifications in the later analysis (Loman et al. 2012). Calculations were performed using the following formula: $\mathrm{WHR}=\left(\sum_{\mathrm{i}=1}^{N} n_{\mathrm{i}}^{2}\right) / N$ where $n$ accounts for the length of the homopolymer and $N$ is the total number of homopolymers in the sequence.

All research was performed following the International Society for Ethnobiology Code of Ethics (2006). Interviews were conducted after agreeing on prior informed consent with the informants. Duplicates of collected sample vouchers were deposited at both the herbarium of the Institute of Traditional Medicine, Muhimbili University of Health and Allied Sciences (ITMH) in Tanzania and Naturalis Biodiversity Center (L) in the Netherlands. Permits for both research and export of collected material were obtained from the Tanzania Commission of Science and Technology (COSTECH), Tanzania Wildlife Research Institute (TAWIRI), Tanzania National Parks Authority (TANAPA) and the Phytosanitary Section of the Ministry of Agriculture and Food Security.

\section{Results}

\section{Clustering thresholds}

Clustering sequencing reads into MOTUs using clustering thresholds lower than most interspecific genetic distances can result in chimeric MOTUs and inaccurate species identification. If reads are clustered into MOTUs based on a $97 \%$ sequence similarity, then 
one assumes that species within the target genus have a sequence similarity of less than $97 \%$, but this might not be the case among recently diverged species. As a consequence, a single MOTU might contain more than one species and thus overlook species diversity in the studied mixture. Pairwise comparisons of the genetic distances of the nrITS sequences of the studied genera were created to determine interspecific variation and the suitability of the clustering criteria for molecular identification (Fig. 1). These pairwise comparisons show the percentage of interspecific genetic distances that exceed the 97 and 99\% MOTU clustering thresholds, and provide a basis for the clustering of amplicons. A low clustering threshold risks creating MOTUs that consist of reads from different species. In this study, some of the main species assessed (Disa spp., Habenaria spp. and Satyrium spp.) have interspecific genetic distances of less than the clustering threshold. For example, only $80.3 \%$ of Habenaria interspecific genetic distances exceed 3\% variation, and thus $19.7 \%$ of distances would fall within the $97 \%$ clustering threshold. The $99 \%$ clustering threshold enhances the discriminatory power for both nrITS1 and nrITS2 for all three genera to at least $94.7 \%$. A clustering threshold of $100 \%$ would have created numerous single or low read number MOTUs splitting species with intraspecific variation into several MOTUs. Moreover, PCR and sequencing errors could cause false MOTUs, potentially a significant issue with high thresholds (i.e., 99-100\%). An increased minimum read number per cluster $(>10)$ could overcome this, but test runs on the Galaxy pipeline with these settings show that this would filter out most of the non-orchid species diversity and thus make the analysis significantly less informative, while removing quite reliable data about non-orchid ingredients (Table 1). As a result, we opted for a 99\% clustering threshold with $>2$ reads per cluster.

\section{Molecular operational taxonomic units and identification}

The Ion-Torrent runs of the six chikanda samples for both markers generated between 8051 and 110,102 raw reads per sample. Clustering and filtering of the reads yielded 41-994 MOTUs per sample and marker. Similarity matching of these MOTUs by localBLAST

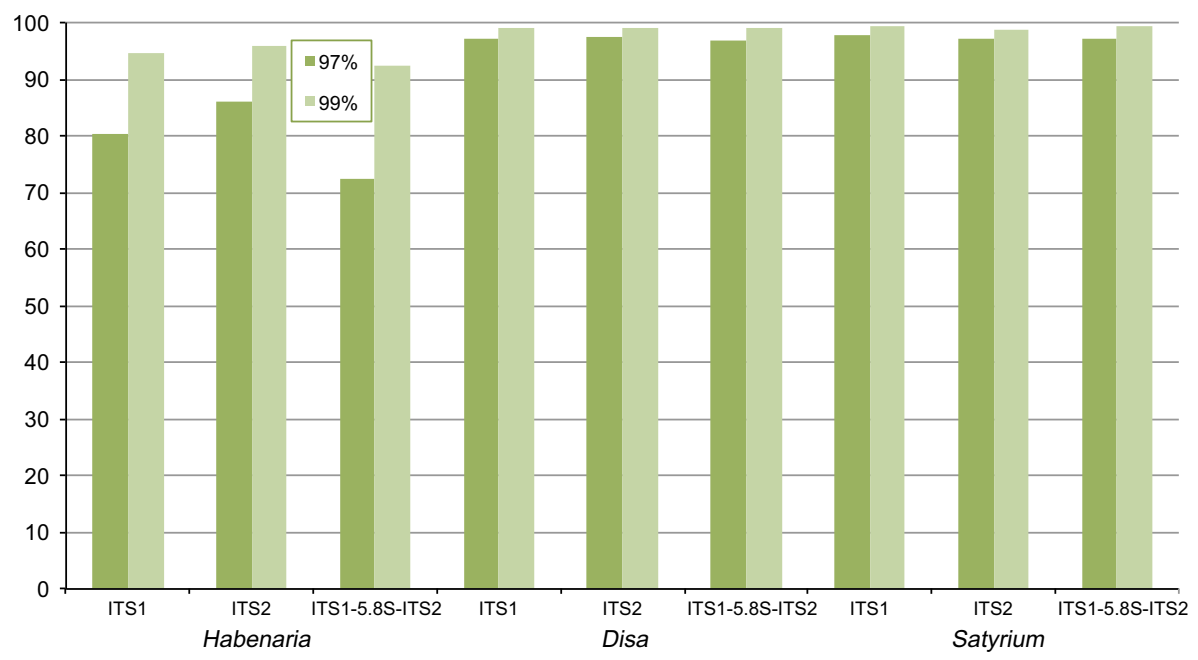

Fig. 1 Percentage of interspecific genetic distances that exceed the 97 and $99 \%$ amplicon clustering thresholds. (Color figure online) 


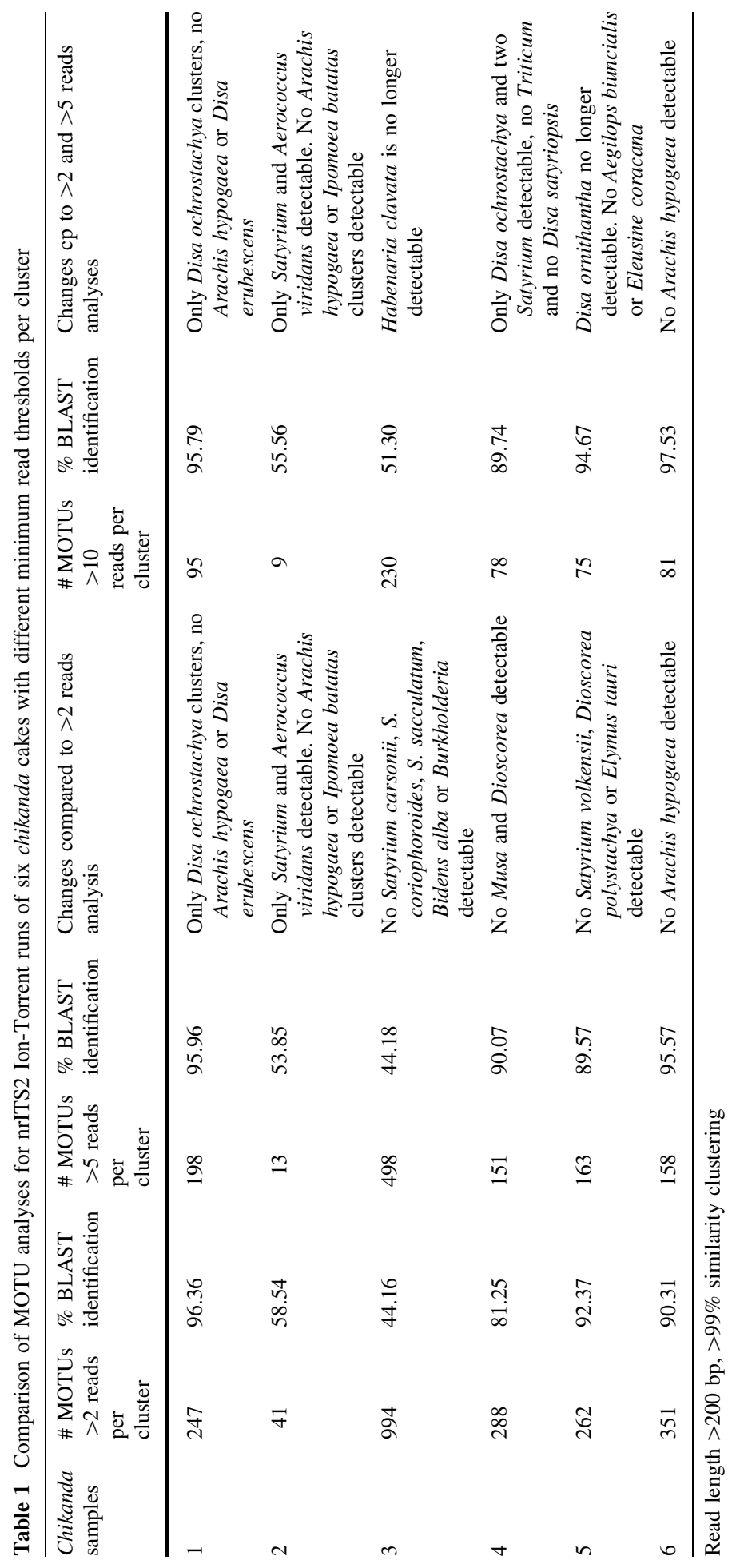


using our tailored sequence reference databases yielded hits for $34.66-88.01 \%$ of the MOTUs (Table 2). The WHR for the three orchid genera did not exceed two, which indicates that it unlikely that homopolymer runs would account for a significant raise in sequencing errors.

\section{Species composition in chikanda}

For nrITS2 a total of 1482 MOTUs were matched using BLAST sequence similarity to 35 plant taxa. The BLAST identifications included at least 17 orchid species: ten Satyrium species, 6 Disa spp., and 1 Habenaria species (Table 3). The combined results for nrITS2 MOTUs from the six chikanda samples show that Disa MOTUs account for 59\% of the reads, Satyrium MOTUs for 30\%, Habenaria MOTUs for just $0.1 \%$ and other MOTUs for another 11\%. Nearly all (99.99-100\%) nrITS1 MOTUs were identified as peanut (Arachis hypogaea). Only two MOTUs, one in chikanda sample 1 and one in sample 4, were identified differently: as Serratia plymuthica, a gram-negative bacterium. No other species were detected using the nrITS marker. The nrITS2 MOTUs yielded primarily orchid hits, but also species from several other families. The separate BLAST run on the African Habenaria database reveals the presence of two additional Habenaria species in chikanda sample 3. All chikanda samples show GenBank hits with Habenaria when we run a local BLAST search with our separate database, but most hits are from fragments of 106 to 116 bp and a sequence identity percentage (97.17-98.11\%). In chikanda 3, however, three MOTUs are identified as Habenaria with a hit length of 341, 343, $341 \mathrm{bp}$ and a sequence identity percentage of $97.95,98.54,99.41 \%$, respectively. Two out of three MOTUs have shared top hits in the BLAST search. One has a shared top hit (341 bp hit length, $99.41 \%$ sequence identity) for Habenaria microsaccos, Habenaria macrostele and Habenaria kyimbilae, and the other a shared top hit (341 bp hit length and $97.95 \%$ sequence identity) for Habenaria tenuispica and Habenaria praestans. The third MOTU is identified as Habenaria clavata (343 bp hit length, $98.54 \%$ sequence identity), but has a close follow-

Table 2 Overview of Ion-Torrent PGM results for nrITS sequences in all six chikanda samples

\begin{tabular}{lcllll}
\hline Sample \# & \# Raw reads & $\begin{array}{l}\text { \% Filtered } \\
\text { reads }\end{array}$ & \# MOTUs & $\begin{array}{l}\text { \# Non singleton } \\
\text { MOTUs }\end{array}$ & $\begin{array}{l}\text { \% BLAST hit } \\
\text { reads (filtered) }\end{array}$ \\
\hline 1 ITS1 & 18,266 & 67.87 & 2346 & 472 & 76.44 \\
1 ITS2 & 30,191 & 31.79 & 1493 & 247 & 84.69 \\
2 ITS1 & 22,846 & 63.07 & 2610 & 555 & 74.05 \\
2 ITS2 & 8051 & 10.91 & 242 & 41 & 50.8 \\
3 ITS1 & 18,668 & 64.01 & 2164 & 480 & 73.37 \\
3 ITS2 & 110,102 & 21.42 & 5815 & 994 & 34.66 \\
4 ITS1 & 23,702 & 64.93 & 2357 & 518 & 78.41 \\
4 ITS2 & 22,975 & 34.39 & 1789 & 288 & 71.63 \\
5 ITS1 & 21,876 & 69.06 & 2328 & 547 & 81.12 \\
5 ITS2 & 24,943 & 57.75 & 2633 & 351 & 80.09 \\
6 ITS1 & 22,313 & 64.47 & 2140 & 500 & 88.01 \\
6 ITS2 & 17,377 & 74.28 & 1456 & 262 & \\
\hline
\end{tabular}

Read length $>200 \mathrm{bp},>99 \%$ similarity clustering, $>2$ reads per cluster 
Table 3 Species identified in chikanda samples from amplicon metabarcoding of MOTUs

\begin{tabular}{|c|c|c|c|}
\hline Ingredients & Families & Common names, usage & Sample \# \\
\hline \multicolumn{4}{|l|}{ Plants } \\
\hline Mangifera indica & Anacardiaceae & Mango, food crop & 3 \\
\hline Bidens spp. & Asteraceae & Traditional medicine & 3 \\
\hline Helianthus spp. & Asteraceae & Sunflower, food crop & 5 \\
\hline Cannabis sativa & Cannabaceae & Marijuana, psycho-active plant & 3 \\
\hline Ipomoea batatas & Convulvulaceae & Sweet potato, food crop & 2 \\
\hline Cucurbita maxima & Cucurbitaceae & Pumpkin, food crop & 3 \\
\hline Cucurbita pepo & Cucurbitaceae & Squash, food crop & 2 \\
\hline Dioscorea polystachya & Dioscoreaceae & Chinese yam, food crop & $4-5$ \\
\hline Arachis hypogaea $^{\mathrm{a}}$ & Fabaceae & Peanut, food crop & $1-6$ \\
\hline Abelmoschus esculentus & Malvaceae & Okra, food crop & 3 \\
\hline Coscinium fenestratum & Menispermaceae & Medicine (SE Asia) & 3 \\
\hline Musa spp. & Musaceae & Banana/plantain, food crop & 4 \\
\hline Disa erubescens & Orchidaceae & Orchid & 1 \\
\hline Disa miniata & Orchidaceae & Orchid & $4-6$ \\
\hline Disa ochrostachya ${ }^{\mathrm{a}}$ & Orchidaceae & Orchid & $1-6$ \\
\hline Disa robusta & Orchidaceae & Orchid & 3 \\
\hline Disa satyriopsis & Orchidaceae & Orchid & $4-6$ \\
\hline Disa aff. similis ${ }^{\mathrm{a}}$ & Orchidaceae & Orchid & 3 \\
\hline Habenaria clavata ${ }^{\mathrm{a}}$ & Orchidaceae & Orchid & 3 \\
\hline Habenaria macrostele ${ }^{\mathrm{a}}$ & Orchidaceae & Orchid & 3 \\
\hline Habenaria praestans $^{\mathrm{a}}$ & Orchidaceae & Orchid & 3 \\
\hline Satyrium anomalum & Orchidaceae & Orchid & 3,5 \\
\hline Satyrium breve & Orchidaceae & Orchid & $2-3$ \\
\hline Satyrium carsonii & Orchidaceae & Orchid & 3 \\
\hline Satyrium comptum & Orchidaceae & Orchid & $4-6$ \\
\hline Satyrium coriophoroides & Orchidaceae & Orchid & 3 \\
\hline Satyrium elongatum & Orchidaceae & Orchid & $2-6$ \\
\hline Satyrium aff. elongatum ${ }^{\mathrm{a}}$ & Orchidaceae & Orchid & 2 \\
\hline Satyrium riparium & Orchidaceae & Orchid & $3-6$ \\
\hline Satyrium shirense & Orchidaceae & Orchid & $4-6$ \\
\hline Satyrium trinerve & Orchidaceae & Orchid & 2,3 \\
\hline Satyrium aff. trinerve ${ }^{\mathrm{a}}$ & Orchidaceae & Orchid & 2 \\
\hline Satyrium volkensii & Orchidaceae & Orchid & 3,5 \\
\hline Eleusine coracana & Poaceae & African finger millet, food & 5 \\
\hline Elymus spp. & Poaceae & Grass & 3,5 \\
\hline Pseudoroegneria spp. & Poaceae & Grass & 3 \\
\hline Triticum aestivum & Poaceae & Common wheat, food crop & 4,5 \\
\hline Capsicum aпnиит & Solanaceae & Chili pepper, food crop & 6 \\
\hline Aquilaria sinensis & Thymelaeaceae & Medicine (SE Asia) & 3 \\
\hline \multicolumn{4}{|l|}{ Bacteria } \\
\hline Burkholderia phytofirmans & Burkholderiaceae & Plant associated bacterium & 3 \\
\hline Klebsiella variicola & Enterobacteriaceae & Human pathogen & 3 \\
\hline
\end{tabular}


Table 3 continued

\begin{tabular}{llll}
\hline Ingredients & Families & Common names, usage & Sample \# \\
\hline Salmonella enterica & Enterobacteriaceae & Human pathogen & 3 \\
Burkholderia cenocepacia & Burkholderiaceae & Plant associated bacterium & 3 \\
Pantoea vagans & Enterobacteriaceae & Plant associated bacterium & 3 \\
Enterobacter cloacae & Enterobacteriaceae & Human gut flora & 3 \\
\hline
\end{tabular}

${ }^{a}$ See discussion for an evaluation of the identifications

up in Habenaria gonatosiphon with a hit length of 345 bp and a sequence identity percentage of $98.26 \%$.

More species were detected in chikanda sample 3 than in any of the other samples (Fig. 2), and this includes 12 non-orchid MOTUs that could be identified as food crops, e.g., Arachis hypogaea (peanut), Abelmoschus esculentus (okra), Mangifera indica (mango), and Cucurbita maxima and C. pepo (squashes and pumpkins), as well as human pathogens (Salmonella spp. and Klebsiella spp.). Detection of Aquilaria sinensis, a southeast Asian sandalwood species that is CITES appendix II listed could be attributed to unintentional contamination from wildlife forensic DNA barcoding research on Aquilaria done in the same lab. For the other samples the number of non-orchid MOTUs identified ranged from one to six, and these were mostly food crops. They included Arachis hypogaea (peanut), Ipomoea batatas (sweet potato), Mangifera indica (mango), Helianthus annuus (sunflower), Eleusine coracana (African pearl millet) and Capsicum annuum (chili pepper).

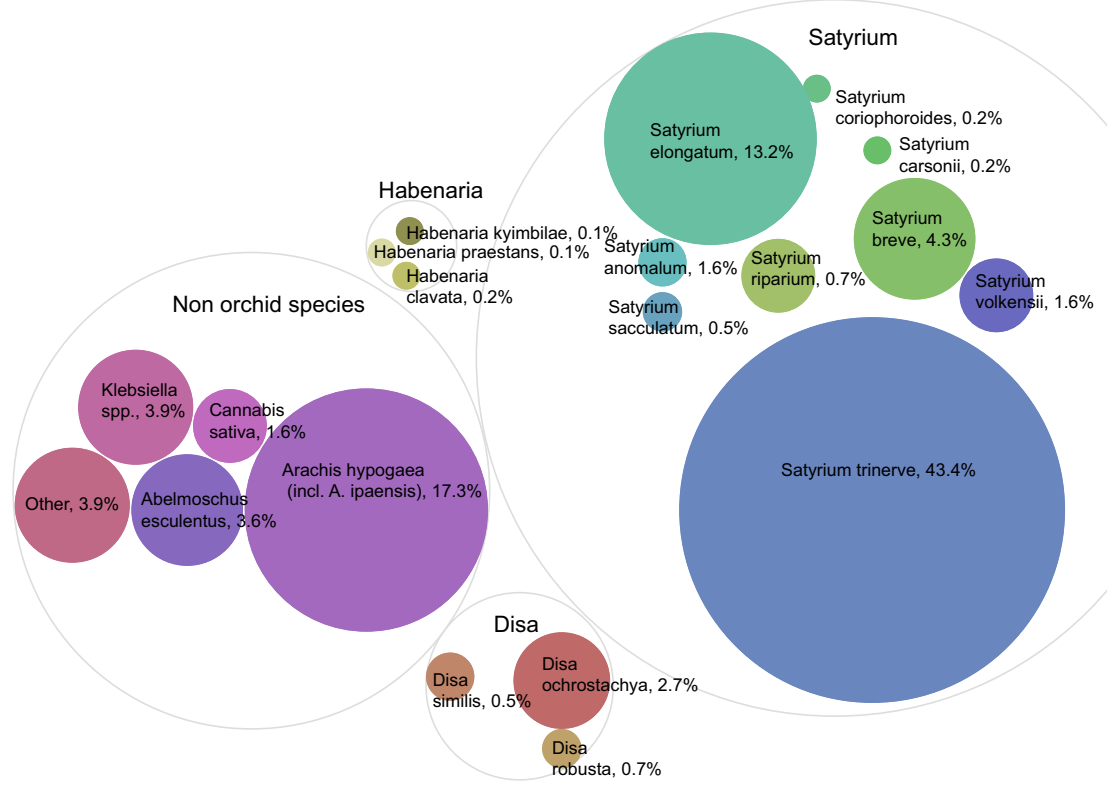

Fig. 2 Species composition of chikanda, expressed as relative abundance of nrITS2 amplicon metabarcoding reads per BLAST identified MOTU for chikanda sample 3. (Color figure online) 


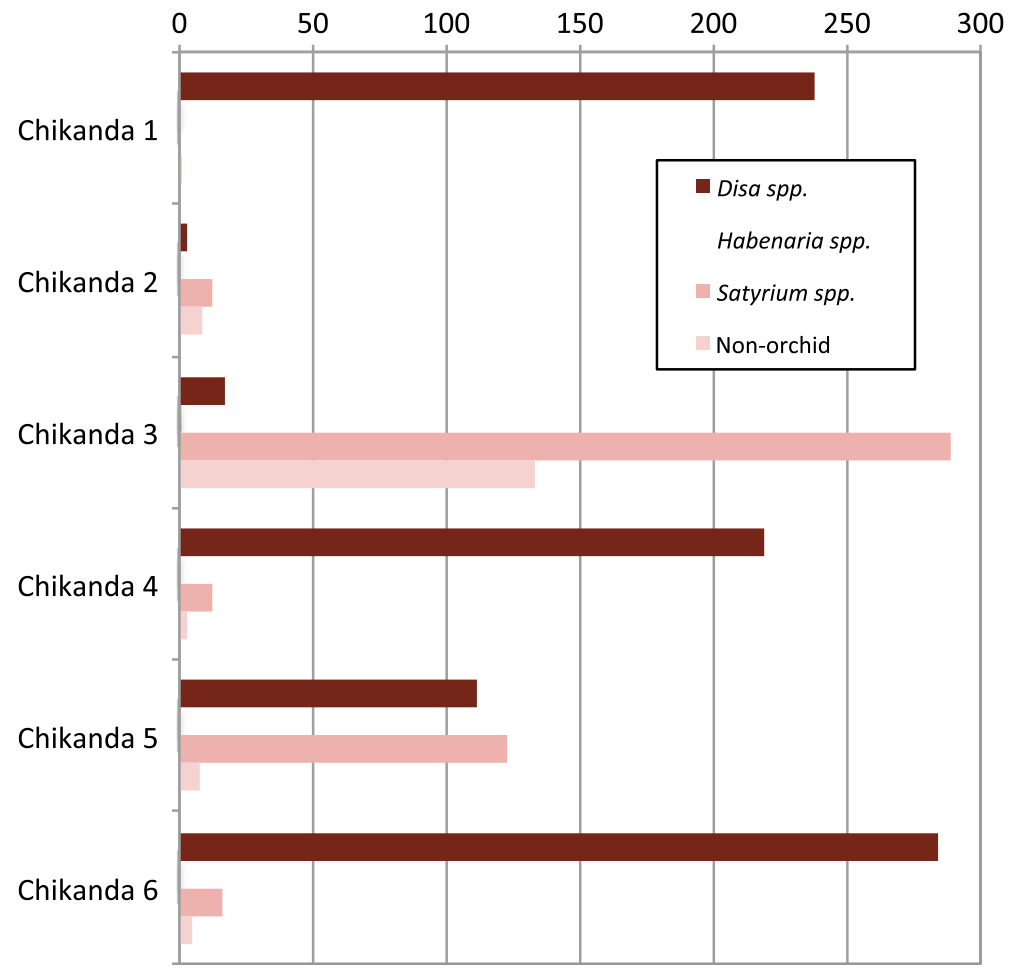

Fig. 3 Genus composition of chikanda samples based on relative abundance of nrITS2 amplicon metabarcoding reads. (Color figure online)

The MOTU composition of chikanda varied among the samples (Fig. 3). The nrITS2 sequences of chikanda 1, for example, only reveal the presence of three MOTUs, which resulted in BLAST identifications for Disa ochrostachya, Disa erubescens and Arachis hypogaea, whereas other samples showed a much higher diversity, ranging from 8 to 27 MOTUs. Besides peanut that was predominant in the nrITS1 reads, Disa MOTUs accounted for most of the reads in chikanda 1, 4, and 6 (99.6, 94 and 93\% of the ITS2 reads, respectively), whereas chikanda 2 and 3 had a larger portion of reads for Satyrium MOTUs (50 and 66\% of ITS2 reads, respectively). Chikanda 5 was the only sample that appeared to have a nearly equal amount of reads for the two main orchid genera, with $51 \%$ Satyrium and $46 \%$ Disa. From all of the chikanda samples only three MOTUs, identified as species of the genus Habenaria, were found, all present in chikanda 3.

\section{Discussion}

The results of the chikanda metabarcoding show that identifications at genus level can be made unambiguously with nrITS2 metabarcoding. Taxa from all three orchid genera have been found in the analyzed chikanda cakes. Disa species were present in all of the samples, Satyrium species in five out of six and Habenaria species in one of the samples. Although a greater diversity of Satyrium species was found in chikanda, reads of Disa species are far greater in number. The Satyrium species identified from the chikanda MOTUs account for 
only $30 \%$ of all reads, whereas the Disa species account for $59 \%$. Additional tests would need to be performed to determine if more reads for Disa species could indicate that Disa species were used in higher quantities or that the higher read number is an artifact of PCR or sequencing bias. Interestingly, when comparing the species composition of chikanda 4 and 6, which were made with 'real' chikanda tubers with the species composition of chikanda 5, which was made with inferior orchid tubers, it seems to support the idea that Disa tubers are preferred and that Satyrium tubers are indeed deemed to be of lower quality, since half of the reads in chikanda 5 were identified as Satyrium species. It should be noted however, that the read numbers cannot be used to quantify the species composition of the chikanda cakes, nor to confirm absence of a species, rather, it is likely to indicate the presence of the species that have been identified. Even though read numbers cannot reliably be used for quantification, the fact that Disa is present in all of the samples and the fact that Disa ochrostachyalDisa robusta is the most commonly identified Disa MOTU, corroborates findings from previous studies in which Disa species were also said to be used preferentially (Richards 1939; Bingham 2004).

MOTUs identified within the genus Habenaria were present in only one of the six chikanda samples. This is far less than expected as various papers dealing with chikanda indicate that the genera Disa, Satyrium and Habenaria are equally affected (Bingham and Smith 2002; Davenport and Ndangalasi 2003; Bingham 2004; Nyomora 2005; Challe and Price 2009). Based on the literature it was expected that Habenaria would be present in chikanda in a similar ratio as Satyrium as a common substitute for the preferred Disa species. The genus Habenaria was well represented in the reference database used for the MOTU identification for both nrITS1 and nrITS2, so underrepresentation is unlikely to have contributed to the low number of Habenaria species found in the chikanda cakes. In addition, poor primer fit and resulting bias is unlikely as the nrITS1 and nrITS2 sequences of Habenaria were contributed by one of the co-authors, and these were amplified with the same primers as used in this study. The tuber morphology of the larger Habenaria species is very similar to that of Satyrium and Disa, which make it unlikely that Habenaria tubers are deemed of lower quality. However, it might be that Habenaria species are targeted less because their inflorescences might break off after flowering and fruiting is over, which would make it more difficult to spot them in the field. The inflorescences of Disa and Satyrium, on the other hand, are sturdier and can be spotted even when the flowers have dried. Another explanation for the prevalence of Disa and Satyrium species above Habenaria species in chikanda cake might be that Disa ochrostachya, D. erubescens, and many of the Satyrium species tend to form colonies of plants, whereas Habenaria species are usually much more scattered. Lastly, it might be possible that Habenaria species are absent from almost all the chikanda samples because collection of chikanda samples took place outside of the peak season.

When we look at the MOTU diversity within the orchid cakes, some intriguing results are found that warrant a closer examination of the identifications. In several of the chikanda samples ambiguous MOTU identifications were found. Disa ochrostachya and Disa robusta were consistently grouped together as the highest BLAST hit, up to an identity match of $99.58 \%$ for a 323 bp hit length, indicating that the resolution for nrITS2 in this clade is too low. The species present in the orchid cake could be either one of them, or both, or a closely related sister species, which has no sequence available in GenBank. Another clade that shows a resolution ambiguity is that containing Satyrium anomalum, $S$. elongatum, S. riparium and $S$. volkensii. MOTUs identified as any of these species usually have very similar scores for the lower ranked BLAST hits, and this could indicate that the species present in the chikanda cake is a closely related species instead. Another 
explanation would be that the individual in the cake is from a disparate population (e.g., from another country) than the individual present in GenBank and that intraspecific distance between those populations result in a seemingly less accurate BLAST identification. Large intraspecific genetic distances and database gaps will lead to less accurate species assignments. All Disa species (except for D. similis) identified in the chikanda samples are represented in GenBank from vouchers collected in Tanzania (Bytebier et al. 2007). For Satyrium, several of the GenBank sequences come from individuals collected in Malawi and South Africa, which could have an effect on the accuracy of the identifications if the query individual and the reference individual are from divergent populations (Van der Niet and Linder 2008). Inspection of the results of Satyrium elongatum and Satyrium trinerve clusters showed distinct molecular signatures in several separate clusters, indicating that, besides Satyrium trinerve and Satyrium elongatum, two closely related species are present in the cake as well (Table 3).

In bench-marking studies, it has been shown that Ion-Torrent PGM reads are prone to indels and specifically insertions (Bragg et al. 2013), but Quail et al. (2012) report that if sequence quality is curated accurately in downstream analyses, then read accuracy on IonTorrent PGM should be similar to that of Illumina MiSeq platforms. However, visual inspection of the reads shows that many sequences appear to include sequencing errors despite the stringent quality control, and when matching query sequences to references, queries often contain anomalous insertions. Stricter quality filtering, however, would most likely result in loss of valuable information of the species composition in mixtures, therefore it seems useful to avoid too strict filtering on quality boundaries, but instead visually evaluate the data.

Lastly, several MOTUs from chikanda 3 match with Disa similis. This is remarkable, because $D$. similis has not been recorded from Tanzania, but so far only from Zambia, Angola and South Africa (La Croix and Cribb 1995). This could mean that the nrITS2 sequence of $D$. similis is very similar to a Tanzanian species not included in our reference database, but it could also indicate that part of the tubers used for this chikanda originated from Zambia, or that $D$. similis occurs in Tanzania but has not yet been recorded by botanists there. The latter possibility is rather unlikely as this is a very distinctive orchid with pale violet to blue flowers. The former possibility seems more probable, since our 99\% clustering threshold can only distinguish $99.19 \%$ of interspecific genetic distances in the reference database, and missing species could also be genetically similar to included species such as $D$. similis. This is supported by the fact that the section Aconitoideae, to which D. similis belongs, has been undersampled in the prevailing Disa phylogeny (Bytebier et al. 2007). Taking this into account it might well be possible that the species in the chikanda sample is one of the sister species to Disa similis, such as D. aperta, D. equestris, D. dichroa or even $D$. nyikensis. These uncertain identifications are a serious indication that we are likely currently underestimating species diversity in chikanda cakes, and we stress the fact that it is essential in this kind of research to have an understanding of the systematics and phylogenetic relationships of the analyzed species.

None of the orchid species identified from these chikanda samples are currently listed on the East-African or global IUCN Red List (2014), but data on most orchid species in this area is deficient. The intensive commercial collection appears to be unsustainable and the need for protection is becoming increasingly urgent. Records from 2001 show that up to 4 million orchid tubers were harvested every year, and a recent survey from 2014 shows amounts have stayed at similar levels, but that orchid tubers are sourced from further afield every year as local populations are depleted (Davenport and Ndangalasi 2001, 2003; Bingham 2004; Veldman et al. 2014). 
In addition to orchids, 17 other plant species and 6 bacterial species (Table 2) were identified among the clustered MOTUs. Two species, Arachis hypogaea and Capsicum annuum are traditional chikanda cake ingredients and were both recovered in the sequencing runs, as expected. Species such as Mangifera indica (mango), Helianthus annuus (sunflower), Ipomoea batatas (sweet potato) and Cucurbita maxima (pumpkin) are all abundantly present in the stalls and sheds were the chikanda is traded, prepared and sold, and can thus be expected to be present in chikanda even if they were not intentionally added. Some MOTU identifications yield unexpected results and this could be due to insufficient reference sequences in GenBank. The identification of Dioscorea polystachya was unexpected as this species is not cultivated locally, but several other Dioscorea species, for which there are no nrITS sequences in GenBank, are grown as tuber crops in Eastern Africa. The identification of Pseudoroegneria spp., Coscinium fenestratum, and Aquilaria sinensis could reflect a similar situation, but above the level of genera. Pseudoroegneria is a genus of North American grass, but the amplified DNA could belong to species from a closely related African genus. The same might apply to C. fenestratum and A. sinensis, which are both southeast Asian medicinal plant species, but might have closely related African genera that did not have nrITS sequences deposited in GenBank at the time of the BLAST identifications. These issues highlight the difficulties posed to species identification using DNA based methods.

Amplification bias in amplicon metabarcoding can have major impacts on the number of reads generated in next generation sequencing for species in mixtures (Pawluczyk et al. 2015). In our study, the majority of nrITS1 amplicon sequencing reads were Arachis hypogaea (peanut) and nrITS2 reads show predominantly orchids, as well as some other species, but almost no Arachis. The Sorghum universal primers (Sun et al. 1994) used in this study have good primer fit for both nrITS1 and nrITS2 for both Arachis and the targeted orchid genera. Nevertheless the amplification and sequencing process can yield a bias in the final number of reads (Pawluczyk et al. 2015), so estimating abundance based on read depth should be treated with caution.

MOTU based identification to species level with nrITS1 and nrITS2 should carefully consider clustering parameters as a low threshold (97\%) could lead to grouping of multiple species into single MOTUs, whereas a high threshold (99\%) could result in oversplitting of MOTUs and loss of valuable data in MOTU filtering. Pairwise comparisons of interspecific genetic distance among species in the orchid genera Disa, Satyrium and Habenaria shows that respectively $97.5,97.1$ and $80.3 \%$ of interspecific distances are less than $3 \%$. An MOTU clustering threshold of 3\% divergence would thus group up to $19.7 \%$ of Habenaria species into single MOTUs. A more stringent threshold of $1 \%$ would cluster up to $5.3 \%$ of Habenaria species into single MOTUs, and only $0.8 \%$ of Disa and $1.4 \%$ of Satyrium species. Studies such as Ivanova et al. (2016) that use a clustering threshold of $2 \%$, risk overlooking species diversity among closely related species in their metabarcoding downstream analyses. Something that needs to be considered as well, however, is sequence inaccuracies due to PCR amplification and sequencing errors. If the clustering threshold used is too stringent erroneous sequences can be grouped in MOTUs and could thus be mistaken for separate taxa. However, the use of High Fidelity DNA polymerases reduces PCR error rates and improves quality filtering in downstream sequence analysis, and removal of singleton MOTUs eliminates large quantities of uncertain or inaccurate reads. In our analyses we found that relaxing the clustering and quality filtering thresholds reduces the risk of formation of MOTUs based on PCR or sequencing errors, but that these less stringent MOTUs underestimate species diversity. The current thresholds yield a higher number of MOTUs, but in the similarity matching we find a large level of 
identification redundancies (i.e., multiple MOTUs matching to the same species). Increasing the number of target genes would greatly reduce the uncertainty in species-level identification.

Lastly, it should be taken into account, that this study used only six chikanda samples, and this sample size makes it difficult to generalize the results and draw conclusions for all commercialized chikanda. When looking at a larger scale, results might deviate from the results in this study and show a higher diversity of orchids used or possibly more adulterated chikanda cakes.

\section{Conclusions}

Amplicon metabarcoding using the barcoding markers nrITS1 and nrITS2 shows the potential advantages of these markers and approach for analysis of species composition, as well as highlight some of the disadvantages. The analysis of interspecific genetic distances between sequences of these markers for the three target orchid genera shows that identifications at generic level can be made with a high level of confidence, but that species-level identifications should be closely examined to minimize identifications based on false assignments to sister taxa that are not represented in GenBank. Data deficiency for orchids is a serious problem in Eastern Africa, and none of the 17 orchid species identified in our samples are currently listed on the East-African or global IUCN Red List (2014). Increased availability of reference sequences in GenBank is likely to improve resolution in species identification and make this approach more widely applicable in the future. For terrestrial orchids used in chikanda this will enable quick assessment of genetic diversity of MOTUs in prepared products and subsequent inference of provenance of the used tubers. Understanding the species diversity and provenance of chikanda orchid tubers will enable the chain of commercialization of these sensitive species to be traced back to the harvesters and their natural habitats, and thus allow for targeted efforts to protect or sustainably use these wild populations.

\section{Supporting information}

Raw sequence read data from the Ion-Torrent PGM is deposited in Dryad in FASTQ format (http://dx.doi.org/10.5061/dryad.tn641). In addition to the FASTQ files the primer sequences for ITS1 and ITS2 (Sun et al. 1994) that were used for demultiplexing and primer trimming are also provided in a csv file. All MOTUs, the number of reads per MOTU and the localBLAST identifications are provided per sample and marker in Appendix S1.

Acknowledgements The authors would like to thank the market vendors and chikanda mamas who participated in the field study. In addition, all authors acknowledge funding from NWO-SIDA-COSTECH TASENE Grant W 02.29.102.

Open Access This article is distributed under the terms of the Creative Commons Attribution 4.0 International License (http://creativecommons.org/licenses/by/4.0/), which permits unrestricted use, distribution, and reproduction in any medium, provided you give appropriate credit to the original author(s) and the source, provide a link to the Creative Commons license, and indicate if changes were made. 


\section{References}

Altschul SF, Gish W, Miller W et al (1990) Basic local alignment search tool. J Mol Biol 215:403-410

Bingham MG (2004) Chikanda trade in Zambia. Orchid Conserv News 4:22-25

Bingham MG, Smith PP (2002) Southern African plant red data lists: Zambia. SABONET, Pretoria

Blaalid R, Kumar S, Nilsson RH et al (2013) ITS1 versus ITS2 as DNA metabarcodes for fungi. Mol Ecol Resour 13:218-224. doi:10.1111/1755-0998.12065

Boessenkool S, Mcglynn G, Epp LS et al (2014) Use of ancient sedimentary DNA as a novel conservation tool for high-altitude tropical biodiversity. Conserv Biol 28:446-455. doi:10.1111/cobi.12195

Bragg LM, Stone G, Butler MK et al (2013) Shining a light on dark sequencing: characterising errors in Ion Torrent PGM data. PLoS Comput Biol 9:e1003031

Bytebier B, Bellstedt DU, Linder HP (2007) A molecular phylogeny for the large African orchid genus Disa. Mol Phylogenet Evol 43:75-90

Challe JF, Price LL (2009) Endangered edible orchids and vulnerable gatherers in the context of HIV/AIDS in the Southern Highlands of Tanzania. J Ethnobiol Ethnomed 5:41. doi:10.1186/1746-4269-5-41

Cheng X, Su X, Chen X et al (2014) Biological ingredient analysis of traditional Chinese medicine preparation based on high-throughput sequencing: the story for Liuwei Dihuang Wan. Sci Rep 4:5147. doi:10.1038/srep05147

CITES (2014) The Convention on International Trade in Endangered Species of Wild Fauna and Flora Appendices. http://www.cites.org/. Accessed 1 May 2016

Coghlan M, Haile J, Houston J et al (2012) Deep sequencing of plant and animal DNA contained within traditional Chinese medicines reveals legality issues and health safety concerns. PLoS Genet 8:e1002657. doi:10.1371/journal.pgen.1002657

Cribb PJ, Leedal GP (1982) The mountain flowers of southern Tanzania: a field guide to the common flowers. AA Balkema, Rotterdam

Davenport TRB, Bytebier B (2004) Kitulo Plateau, Tanzania-a first African park for orchids. Orchid Rev 112:161-165

Davenport TRB, Ndangalasi HJ (2001) Orchid harvest-an assessment of the harvesting and trade of orchid tubers across Tanzania's Southern Highlands. Wildlife Conservation Society

Davenport TRB, Ndangalasi HJ (2003) An escalating trade in orchid tubers across Tanzania's Southern Highlands: assessment, dynamics and conservation implications. Oryx 37:55-61

De Beeck MO, Lievens B, Busschaert P et al (2014) Comparison and validation of some ITS primer pairs useful for fungal metabarcoding studies. PLoS ONE 9:e97629. doi:10.1371/journal.pone.0097629

De Boer HJ, Ouarghidi A, Martin G et al (2014) DNA barcoding reveals limited accuracy of identifications based on folk taxonomy. PLoS ONE 9:e84291. doi:10.1371/journal.pone.0084291

Doyle JJ, Doyle JL (1987) A rapid DNA isolation procedure for small quantities of fresh leaf tissue. Phytochem Bull 19:11-15

Epp LS, Boessenkool S, Bellemain EP et al (2012) New environmental metabarcodes for analysing soil DNA: potential for studying past and present ecosystems. Mol Ecol 21:1821-1833. doi:10.1111/j. 1365-294X.2012.05537.x

Ewing B, Green P (1998) Base-calling of automated sequencer traces using phred. II. Error probabilities. Genome Res 8:186-194

Galimberti A, De Mattia F, Bruni I et al (2014) A DNA barcoding approach to characterize pollen collected by honeybees. PLoS ONE 9:e109363. doi:10.1371/journal.pone.0109363

Hamisy CW (2008) Development of conservation strategies for the wild edible orchid in Tanzania. Progress report for the Rufford Small Grants Foundation. www.ruffordsmallgrants.org/files/ orchidreport202007[1].doc. Accessed 1 May 2016

Hedberg O (1957) Afroalpine vascular plants: a taxonomic revision. Symb Bot Ups 15:1-411

ISE (2006) International Society of Ethnobiology code of ethics (with 2008 additions). http://ethnobiology. net/code-of-ethics/. Accessed 1 May 2016

IUCN SSC (2014) IUCN Red List of threatened species. Version 2014.3, 2014.3 edn. IUCN, The World Conservation Union, Gland

Ivanova NV, Kuzmina ML, Braukmann TW et al (2016) Authentication of herbal supplements using nextgeneration sequencing. PLoS ONE 11:e0156426. doi:10.1371/journal.pone.0156426

Jørgensen T, Kjaer KH, Haile J et al (2012) Islands in the ice: detecting past vegetation on Greenlandic nunataks using historical records and sedimentary ancient DNA meta-barcoding. Mol Ecol 21:1980-1988. doi:10.1111/j.1365-294X.2011.05278.x

Kasulo V, Mwabumba L, Munthali C (2009) A review of edible orchids in Malawi. J Hortic For 1:133-139

Katoh K, Misawa K, Kuma K, Miyata T (2002) MAFFT: a novel method for rapid multiple sequence alignment based on fast Fourier transform. Nucleic Acids Res 30:3059-3066 
Kool A, de Boer HJ, Krüger Å et al (2012) Molecular identification of commercialized medicinal plants in Southern Morocco. PLoS ONE 7:e39459. doi:10.1371/journal.pone.0039459

La Croix I, Cribb PJ (1995) Flora Zambesiaca. Orchidaceae. Parts I and II, vol 11. Balkema, Rotterdam

Lammers Y, Peelen T, Vos RA, Gravendeel B (2014) The HTS barcode checker pipeline, a tool for automated detection of illegally traded species from high-throughput sequencing data. BMC Bioinform 15:44. doi:10.1186/1471-2105-15-44

Larsson A (2014) AliView: a fast and lightweight alignment viewer and editor for large datasets. Bioinformatics 30:3276-3278. doi:10.1093/bioinformatics/btu531

Li W, Godzik A (2006) Cd-hit: a fast program for clustering and comparing large sets of protein or nucleotide sequences. Bioinformatics 22:1658-1659

Loman NJ, Misra RV, Dallman TJ et al (2012) Performance comparison of benchtop high-throughput sequencing platforms. Nat Biotechnol 30:434-439

Meier R, Shiyang K, Vaidya G, Ng PKL (2006) DNA barcoding and taxonomy in Diptera: a tale of high intraspecific variability and low identification success. Syst Biol 55:715-728. doi:10.1080/ 10635150600969864

Nyomora AMS (2005) Distribution and abundance of the edible orchids of the Southern Highlands of Tanzania. Tanzan J Sci 31:45-54

Otieno J, Abihudi S, Veldman S et al (2015) Vernacular dominance in folk taxonomy: a case study of ethnospecies in medicinal plant trade in Tanzania. J Ethnobiol Ethnomed. doi:10.1186/1746-4269-1110

Parducci L, Jørgensen T, Tollefsrud MM et al (2012) Glacial survival of boreal trees in Northern Scandinavia. Science 335:1083-1086. doi:10.1126/science.1216043

Pawluczyk M, Weiss J, Links MG et al (2015) Quantitative evaluation of bias in PCR amplification and next-generation sequencing derived from metabarcoding samples. Anal Bioanal Chem 407:1841-1848

Quail MA, Smith M, Coupland P et al (2012) A tale of three next generation sequencing platforms: comparison of Ion Torrent, Pacific Biosciences and Illumina MiSeq sequencers. BMC Genomics 13(1): 1

Richards AI (1939) Land, labour and diet in Northern Rhodesia: an economic study of the Bemba tribe. Oxford University Press, Oxford

Richardson RT, Lin C-H, Sponsler DB et al (2015) Application of ITS2 metabarcoding to determine the provenance of pollen collected by honey bees in an agroecosystem. Appl Plant Sci 3:apps.1400066. doi:10.3732/apps.1400066

Rohland N, Hofreiter M (2007) Ancient DNA extraction from bones and teeth. Nat Protoc 2:1756-1762. doi:10.1038/nprot.2007.247

Samarakoon T, Wang SY, Alford MH (2013) Enhancing PCR amplification of DNA from recalcitrant plant specimens using a trehalose-based additive. Appl Plant Sci 1:apps.1200236. doi:10.3732/apps.1200236

Schmidt P-A, Bálint M, Greshake B et al (2013) Illumina metabarcoding of a soil fungal community. Soil Biol Biochem 65:128-132. doi:10.1016/j.soilbio.2013.05.014

Schmieder R, Edwards R (2011) Quality control and preprocessing of metagenomic datasets. Bioinformatics 27:863-864

Sun Y, Skinner DZ, Liang GH, Hulbert SH (1994) Phylogenetic analysis of Sorghum and related taxa using internal transcribed spacers of nuclear ribosomal DNA. Theor Appl Genet 89:26-32

Taberlet P, Coissac E, Pompanon F et al (2012) Towards next-generation biodiversity assessment using DNA metabarcoding. Mol Ecol 21:2045-2050. doi:10.1111/j.1365-294X.2012.05470.x

Van der Niet T, Linder HP (2008) Dealing with incongruence in the quest for the species tree: a case study from the orchid genus Satyrium. Mol Phylogenet Evol 47:154-174

Van der Niet T, Linder HP, Bytebier B, Bellstedt DU (2005) Molecular markers reject monophyly of the subgenera of Satyrium (Orchidaceae). Syst Bot 30:263-274

Veldman S, Otieno JN, van Andel T et al (2014) Efforts urged to tackle thriving illegal orchid trade in Tanzania and Zambia for chikanda production. TRAFFIC Bull 26:47-50

Willerslev E, Davison J, Moora M et al (2014) Fifty thousand years of Arctic vegetation and megafaunal diet. Nature 506:47-51. doi:10.1038/nature12921 